\title{
Massachusetts Parents' Experiences With Insurance Coverage for Lactation Consultant Services
}

\author{
Robin Snyder-Drummond, BA, IBCLC, RLC ${ }^{1}$ \\ Heather Bingham, BA, IBCLC, RLC ${ }^{2}$ \\ Jessica M. Lang, PhD, IBCLC, RLC, $\mathrm{MPH}^{3}$
}

Share this:

\begin{abstract}
The Women's Preventive Services component of the Affordable Care Act (ACA) requires coverage of "lactation supplies and services" with no out-of-pocket cost to the patient. However, as IBCLCs providing in-home lactation consults, we found that during the first year of the ACA mandate, clients often described disappointment with coverage for home visits and for breastfeeding support in general. To have a more specific picture of women's experiences, our association conducted an online survey of families in Massachusetts who sought insurance coverage for IBCLC care outside of the hospital since the ACA provisions came into effect. The goal was to identify the barriers that families who need these services commonly dealt with. Barriers reported by survey respondents included confusing and inconsistent information provided by insurance companies about coverage, lack of coverage for the time and place they sought IBCLC services, lack of coverage for IBCLCs at all, and restriction to a network that did not include IBCLC providers. These findings suggest that many women are not yet receiving the benefits of insurance coverage for lactation support and point to specific changes that insurance providers should make to provide adequate coverage as mandated under the ACA.
\end{abstract}

Keywords: breastfeeding; Affordable Care Act; insurance

The Women's Preventive Services component of the Affordable Care Act (ACA) requires coverage of "lactation supplies and services" with no out-ofpocket cost to the patient. The Health Resources and Services Administration (n.d.) specifies in the Women's Preventative Services Guidelines that this coverage should include "comprehensive lactation support and counseling, by a trained provider during pregnancy and/ or in the postpartum period."

The National Breastfeeding Center (NBFC) and the United States Breastfeeding Committee (USBC) published Model Policy: Payer Coverage of Breastfeeding Support and Counseling Services, Pumps and Supplies to provide an evidence-based guide to appropriate coverage for breastfeeding support (USBC, NBFC, 2014). However, as IBCLCs providing in-home lactation consults, we found that clients often described disappointment with coverage for home visits, and for breastfeeding support, in general.

A 2013 article by The New York Times described how "despite the law, many new mothers have found it nearly impossible to get timely help for breastfeeding problems"

1. robin@birthready.com

2. heather.bingham@gmail.com

3.jlk916@mail.harvard.edu
(Saint Louis, 2013, para. 3, p. D6). To have a clearer picture of parents' experiences, our association conducted an online survey of families in Massachusetts who sought insurance coverage for IBCLC care outside of the hospital since the ACA provisions came into effect. The goal was to identify the barriers to insurance coverage.

\section{Method}

\section{Survey Development}

The online survey, developed using Formsite, was conducted anonymously. The survey asked respondents about their experiences with insurance coverage since January 2013. This was a mixed-methods study, composed of 10 questions, both closed-and open-ended. The questions, listed in the Appendix, asked about the reasons for seeking IBCLC support, settings of care, and their insurance coverage, with space for further comments. The goal was to gauge the effectiveness of insurance coverage under the terms of the ACA.

We disseminated the survey link broadly and did not expect the respondents to be a representative sample of any population. This survey was posted on our association's website (http://www.lchomevisits.com), on our individual business websites and Facebook pages, and in social media. In addition, we shared the link with clients, colleagues, and other providers who worked with childbearing women. 


\section{Results}

Responses were collected from June 2013 to August 2015. During that time, 72 unique respondents completed the survey. All but one respondent reported having commercial (private) health insurance. The one exception was coverage by TriCare. (After this survey was completed, TriCare updated their policy to cover breastfeeding support.) Eight commercial insurance companies were represented. The three largest companies covered $81 \%$ of the respondents. No recipients of MassHealth, this state's Medicaid program, participated in the survey.

Most respondents (95\%) did see an IBCLC. The majority (75\%) said that they were seen in their homes, and 26\% were seen in an office setting. However, 18 (25\%) said that they delayed seeing an IBCLC because of concerns about insurance coverage. Three of those reported that they did not see an IBCLC at all. (Respondents were allowed to give more than one answer so the totals do not add up to $100 \%$.)

As shown in Figure 1, the most common reasons given for seeking help were pain, concern about baby's low weight gain, or low milk production. Recommendation of a primary healthcare provider for IBCLC care was cited by $38 \%$ of the respondents.

\section{Outcome of Insurance Claims}

Of the 69 respondents who did see an IBCLC, 14 (19\%) reported that they paid out of pocket and never filed for reimbursement. Of those who did file a claim, only $13(24 \%)$ said that they were fully covered, with no cost sharing. Eight respondents received full reimbursement for IBCLC home visits. One insurance company covered those eight.

Those who received full coverage were more likely to have been seen in an office setting (43\%, compared to
$26 \%$ of the total sample). The outcomes of claims are shown in Figure 2.

Half of the respondents reported a claim denial. The most common reason given for denial was that IBCLC services were not covered at all. Respondents also reported restrictions on setting, timing, and network-only providers. Some respondents indicated that these restrictions left them with no access to lactation support at all.

[Insurance company] only covers service by $\mathrm{MD}$ or $\mathrm{RN}$ in office. This was not an option for me as I needed assistance immediately and the wait was several days for a LC at my doctor's office.

They made it specifically difficult to determine whether my provider was in network or to find one who is, and with absolutely no coverage out of network, this effectively rendered my coverage unusable. I am furious that they are allowed to claim they cover this service when they cannot offer a list of providers.

\section{Benefit Information From Insurance Companies}

More than half the respondents (64\%) said that they contacted their insurance company prior to seeing an IBCLC to ask about their coverage. Those who did were asked, "What were you told?" Of these, only 23\% reported that they were told they were covered, with no cost sharing, for IBCLC support (see Figure 2). Some were told they were not covered at all. Thirty percent were told they were covered only if the provider was a doctor or nurse practitioner. Of the eight respondents who were required to see an in-network provider, only three were given a list of providers.

In open responses, confusion and inconsistency emerged as common themes. Respondents commented that they called multiple times and were given different information on different calls. This was often confusing.

\section{Figure 1. Reasons for seeking IBCLC care.}

$$
\begin{array}{r|c}
\text { Inadequate weight gain or milk production }(\mathrm{n}=43) & 60 \% \\
\text { Nipple pain }(\mathrm{n}=39) & 54 \% \\
\text { Baby not latching }(\mathrm{n}=35) & 49 \% \\
\text { Recommendation of my pediatrician or ObGyn/MW }(\mathrm{n}=27) & 38 \% \\
\text { Breast pain }(\mathrm{n}=20) & 28 \% \\
\text { Baby was fussy or uncomfortable }(\mathrm{n}=13) & 18 \% \\
\text { Concerns about feeding pattern or schedule }(\mathrm{n}=12) & 17 \% \\
\text { Mastitis/thrush/infection of nipple or breasts }(\mathrm{n}=8) & 11 \% \\
\text { Other }(\mathrm{n}=14) & 19 \%
\end{array}
$$


Figure 2 . When you contacted insurance, what were you told?

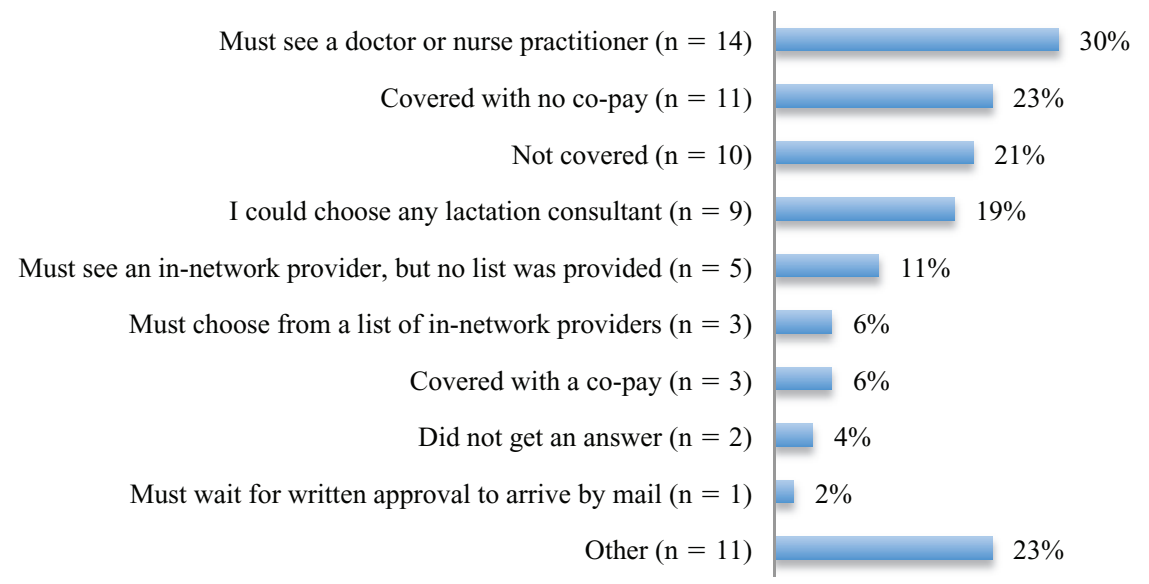

Women reported they were required to see someone within the network then learned there were no IBCLCs in network.

I had to call a few times before getting the right info. At first they just referred me to join La Leche League ... but then clarified that I was covered for lactation services in the hospital after giving birth and could see a doctor or nurse practitioner in a doctor's office and have the service covered. The pediatrician was the one who recommended I see [a lactation consultant].

\section{Gaps in Coverage: Timing and Providers}

All respondents were asked, "Were you able to find an IBCLC who met your insurance company's requirements?" More than half (58\%) said they were not. Reasons included lack of IBCLCs in network, lack of coverage for any IBCLC (sometimes attributed to the lack of a state license), or ignorance of the IBCLC role and credential. Respondents were frustrated to be referred to an IBCLC by a physician, only to be told by their insurance company that they should receive their lactation support from a physician.

When I spoke with an agent . . I was told IBCLC services were not covered. Rather, according to them, under the Affordable Care Act, "lactation counseling" would be covered if provided by a MD or NP.

[Insurance company] said lactation consultants weren't covered bc they aren't medical enough-like they don't have the right degree such as doctor nurse etc. Since I had just had a baby I wasn't really able to do the research to find out they were wrong. Now I'm mad.
Coverage was often restricted to the hospital stay or the first weeks of early postpartum. There is no consensus on how many weeks define postpartum.

Six weeks is not nearly enough time for coverage. I used nipple shields for four months, plus returning to work and pumping. All raise concerns/questions for nursing moms who could use lactation support.

\section{In Their Own Words}

In the personal remarks, respondents had negative words to describe their experience with the insurance process. They used words such as furious, difficult, impossible, incredibly frustrating, and mad and characterized insurance company's denial of support as outrageous and almost criminal.

Many described the vulnerability of the postpartum period. They complained that the insurance process required time and energy that they did not have. Some felt insurance companies were taking advantage of this, counting on families not to appeal. One respondent commented, "Did not have time to deal with this right after baby was born. I saw any IBCLC I could and dealt with insurance after." Another remarked, "It is so hard to get and they take advantage of the fact that you have a small infant and do not have time for their crap."

Many expressed confusion and frustration with the outof-pocket costs. Some respondents delayed or declined services. Others paid out of pocket to expedite care. Those who had paid out of pocket described it as a worthwhile investment. They expressed concern for 
families who could not afford to pay. "These visits are pricey and not everyone can afford it."

I was very disappointed that lactation services were not covered by my insurance! It seems almost criminal!!! But my IBCLC was wonderful and though it was a stretch for us financially, I definitely feel it was worthwhile to pay out of pocket for her help.

Mothers also described the value of IBCLC help, unprompted, in open responses. Several commented that IBCLC support made a crucial difference.

My LC and her support was probably literally life saving for my baby and sanity saving for me. Because of her I breastfed my baby for two years!!! And, he became healthy. It is/was OUTRAGEOUS that [insurance] wouldn't pay for these services. I know that in better times, when I wasn't so overwhelmed, sick, exhausted, and anxious over a sick baby, I would have tried to fight it out a bit more. But instead I took the easier path of just paying out of pocket several times. I know that's exactly what they counted on ... and I'm sorry for that.

Lactation support is what finally helped me to nurse my son effectively. Five months later, and we are still breastfeeding because of the care I received from my lactation visit. I would not have been able to continue breastfeeding otherwise.

\section{Discussion}

These findings demonstrate that not all families are receiving appropriate coverage for IBCLC support under the ACA. Families are confused by inconsistent information and have difficulty understanding what is covered. For the families who responded to our survey, major barriers included:

- Confusing and inconsistent information provided by insurance companies about coverage,

- Lack of coverage for the time and place they sought IBCLC services,

- Lack of coverage for IBCLCs at all, and

- Restriction to a network that did not include IBCLC providers.

At this crucial time, navigating their insurance policy was difficult for families. Confusion leads to delays in getting help and in some cases an inability to access services at all. It is concerning that most respondents sought IBCLC help because of pain, concerns about weight gain, or low milk production. These are all time-sensitive indications with implications for adequate breastfeeding and baby's well-being. These concerns should be a priority.

Inadequate support can lead to early weaning and thus impact baby's optimal nutrition. Mothers are at risk for plugged ducts and mastitis. Left unaddressed, continuing pain can put her at risk for postpartum depression. Kendall-Tackett (2007) has shown definite links between pain, inflammatory response, and postpartum depression. This compounds the problems of breastfeeding and makes the family's adjustment more difficult.

In 2011, The Surgeon General's Call to Action to Support Breastfeeding specifically stated IBCLC credential as the standard for care (U.S. Department of Health and Human Services, 2011). Yet, a lack of recognition for this credential affects insurance coverage.

The NBFC's Model Policy describes approved lactation care providers. Although the IBCLC credential qualifies, it is not specifically mentioned. The Model Policy's list of permitted providers includes medical doctor (MD), advanced practice registered nurse (APRN), doctor of osteopathic medicine (DO), physician assistant (PA), registered nurse $(\mathrm{RN})$, certified childbirth educators, and registered dietitians (registered dietitian [RD], registered dietician nutritionist [RDN], licensed dietician nutritionist[LDN]). However, none of these providers have as thorough a background in lactation education as IBCLCs. Acknowledging IBCLCs as specialists in lactation would reduce the payer's confusion about this support.

Because an IBCLC is not a licensed provider, there is usually no coverage for Medicaid recipients. (Only Georgia and Rhode Island have licensure for IBCLCs.) For low-income mothers on Medicaid plans, this means they may forego appropriate care. This increases the likelihood of early weaning and thus contributes to longer term health issues. Limiting IBCLC support aggravates existing health disparities.

Recently the federal government changed its Medicaid policy to allow IBCLCs to bill for services. However, there are still limitations. Herold and Bonuck (2016) found that few significant changes have resulted in expanded coverage of IBCLCs, especially in states without Medicaid expansion.

Of the 15 states responding, 9 had Medicaid expansion. None of the states permitted IBCLCs to bill for services autonomously. In nine states, IBCLC services were covered with some type of stipulation, for example, 
billing under a physician. Of the nine states with IBCLC coverage, seven had accepted Medicaid expansion.

It should be noted that IBCLC coverage, if provided, did positively influence rates of exclusive breastfeeding. "States with higher rates of exclusive breastfeeding were also more likely to provide IBCLC coverage."

In a review of breastfeeding services and support under the ACA for families on Medicaid and Special Supplemental Nutrition Program for Women, Infants and Children (WIC), Hawkins, Dow-Fleisner, and Noble (2015) found that low-income families receive less adequate support than their peers with more income. The authors noted that

... the present mix of state and federal law... presents an unacceptable patchwork rather than a unified commitment to eliminating breastfeeding disparities. ... . To keep the trend of broadening coverage continuing, both state and federal legislative and regulatory efforts, along with a commitment by insurers to effective implementation of the law, will be needed.

Medicaid's coverage for breastfeeding support is limited and uneven. For private insurance, policies are also inconsistent. Our survey responses echo the findings of the NBFC (2015) annual "Payer Scorecard" report. Drawing from insurance company's published data, each payer was assigned a grade relative to the NBFC Model Payer Policy components of complete coverage for lactation care. That includes:

- Types of services covered,

- Types of provider covered,

- Type of pumps covered,

- Criteria or restriction on coverage, and

- Accessibility.

These scorecards show how widely insurance policies vary. Some companies covered the bare minimum required to comply with the law: a manual hand pump and care rendered by in-network providers (who may have no training in lactation care). Other policies covered fully qualified lactation care providers and the purchase or rental of more effective pumps. The definition of breastfeeding support is inconsistent.

Of the three insurance providers mentioned most often (10 or more respondents) in our survey, all changed their Scorecard grade between 2013 and 2015. One rose from a $\mathrm{D}+$ to a $\mathrm{C}$, one from a $\mathrm{B}-$ to $\mathrm{B}+$, whereas one sank from a $\mathrm{B}+$ to a $\mathrm{C}+$. It is not clear if the changes were because of more or less restriction on coverage or other components of care.

This survey began in 2013, when the Affordable Care Act was fairly new. Families and payers were perhaps unfamiliar with the law. However, the survey was open until 2015, and in that time, there was no discernible improvement in access to IBCLC care observed through the respondents' answers.

From the inception of the ACA, there have been published guidelines for insurance companies to follow. Since 2013, and still ongoing, breastfeeding advocacy groups have been informing the public and promoting the benefits under the new healthcare law. The National Women's Law Center (2014) promotes their Fact Sheet and Tool Kit to understand benefits under the ACA law. The USBC has created and circulated online petitions about coverage for pumps and related issues. While writing this article, we came upon a class action suit that is being filed by Chimicles and Tikellis, LLP (n.d.), in Pennsylvania alleging that the inadequate insurance coverage mothers have experienced is a legal violation.

\section{Limitations of This Survey}

This was a population of less than 100 families, and not representative of the population of Massachusetts. The survey format and methods for disseminating it may have selected for mothers who use the internet, are comfortable English speakers, had been clients of one of the LC Home Visits IBCLCs, had an experience they wished to share, and had the energy to relive a stressful postpartum experience.

Our survey focused only on Massachusetts. This could be expanded to include other states. Our publicity for the survey could be spread further and include formats that do not require computer access. Allowing for more personal remarks and follow-up could reveal further details about appeals or strategies for payment.

All respondents had commercial insurance. This likely reflects the bias created by reaching out most directly to mothers who were clients of private-practice IBCLCs. Surveying families covered by Mass Health (Medicaid) would provide more crucial insights about the experiences of lower income families.

\section{Conclusion}

Our survey identified substantial gaps in coverage for IBCLC support for the responding Massachusetts families. Structural and policy changes are needed to 
address these barriers. Specifically, recognition of the IBCLC role is essential. The Model Policy would be improved by citing the specific standards of the IBCLC.

In The Surgeon General's Call to Action to Support Breastfeeding, Action 11 states "Ensure access to services provided by IBCLCs" and specifies that insurance should "provide reimbursement for IBCLCs independent of their having other professional certification or licensure [emphasis added]."

State licensure for IBCLCs may be one way to assure insurance coverage. Enactment of regulatory requirements for insurance coverage to include IBCLCs could improve access.

Payers need to develop clear policies that provide meaningful coverage. IBCLC providers should be included in the network and in sufficient numbers and locations. Families should not experience delays in care because of a burdensome process for finding an IBCLC. Insurance coverage should allow for the full duration of breastfeeding.

The ACA defines breastfeeding care as preventative; to be effective, it must also be defined as urgent and essential. Clear reassurance that qualified care with an IBCLC is fully covered would be a genuine benefit to families.

\section{References}

Chimicles \& Tikellis, LLP. (n.d.). Complaints filed against insurance carriers for failing to provide ACA-mandated coverage for lactation services. Retrieved from https://chimicles.com/complaintsfiled-insurance-carriers-failing-provide-aca-mandated-coveragelactation-services/
Hawkins, S., Dow-Fleisner, S., \& Noble, A. (2015). Breastfeeding and the Affordable Care Act. Pediatric Clinics of North America, 62, 1071-1091.

Health Resources and Services Administration. (n.d.). Women's preventive services guidelines. Retrieved from https://www.hrsa .gov/womensguidelines/\#

Herold, R., \& Bonuck, K. (2016). Medicaid IBCLC service coverage following the Affordable Care Act and the Center for Medicare and Medicaid Services update. Journal of Human Lactation, 32(1), 89-94. http://dx.doi.org/10.1177/0890334415599164

Kendall-Tackett, K. (2007). A new paradigm for depression in new mothers: The central role of inflammation and how breastfeeding and anti-inflammatory treatments protect maternal mental health. International Breastfeeding Journal, 2, 6. Retrieved from https://internationalbreastfeedingjournal .biomedcentral.com/articles/10.1186/1746-4358-2-6

National Breastfeeding Center. (2015). Payer scorecard. Retrieved from http://www.nbfcenter.com/payer-scorecard.html

National Women's Law Center. (2014). New benefits for breastfeeding moms: Facts and tools to understand your coverage under the Health Care Law. Retrieved from http://nwlc.org/resources/newbenefits-breastfeeding-moms-facts-and-tools-understand-yourcoverage-under-health-care-law/

Saint Louis, C. (2013, September 30). Breast-feeding services lag behind the law. The New York Times. Retrieved from http://nyti .ms/1cUIQH4

United States Breastfeeding Committee, National Breastfeeding Center. (2014). Model policy: Payer coverage of breastfeeding support and counseling services, pumps and supplies (2nd Rev. ed.). Washington, DC: Author.

U.S. Department of Health and Human Services. (2011). The surgeon general's call to action to support breastfeeding. Washington, DC: Author. Retrieved from https://www.surgeongeneral.gov/ $\underline{\text { library/calls/breastfeeding/ }}$

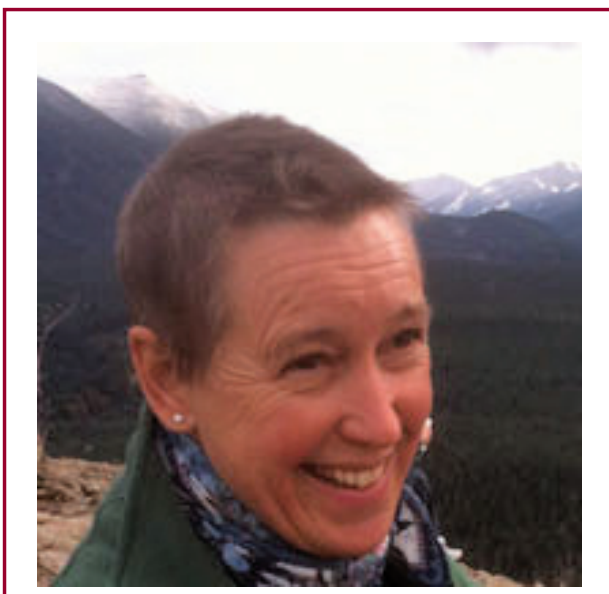

Robin Snyder-Drummond, BA, IBCLC, RLC, has been working with pregnant women and their families since 1993. Her experience as a childbirth educator and doula inspired her to become an IBCLC. Certified since 2009, Robin has been focusing on home visits for breastfeeding guidance. She also teaches breastfeeding in two local hospitals; c-facilitates a Baby Cafe in Dorchester, Massachusetts, and is an active member of LC Home Visits. Her website is birthready.com. 

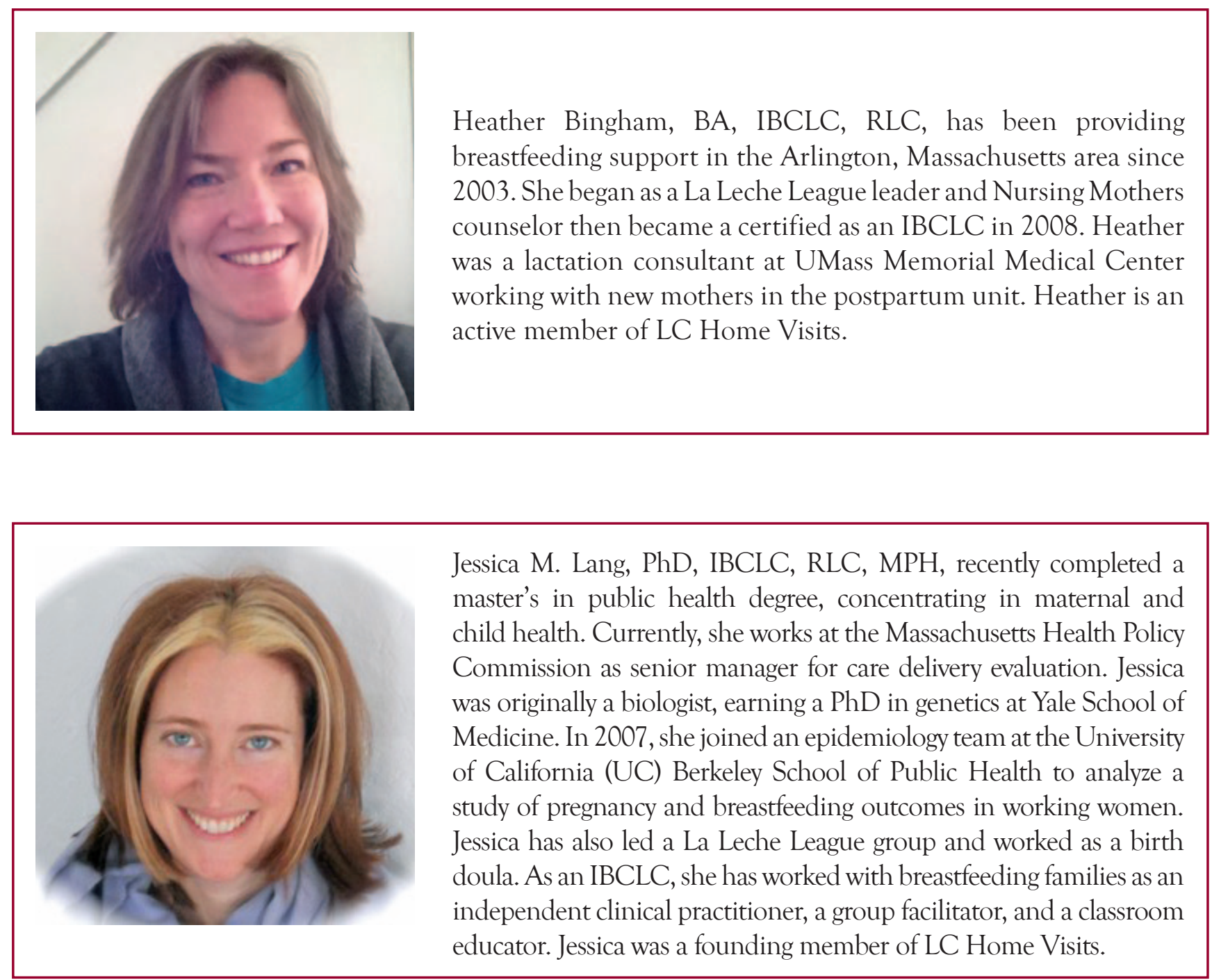

\section{Appendix. Survey Questions}

- Do you live in Massachusetts?

- In what year did you first contact your insurance company or submit a claim for lactation support?

1. When you sought breastfeeding/lactation help outside of the hospital, what was the reason? (check all that apply)

2. In what setting, outside of the hospital, did you see a lactation consultant (IBCLC)?

3. Did you contact your insurance company prior to seeing a lactation consultant to ask about your coverage for lactation support?

4. When you contacted your insurance, what were you told?
5. Were you able to find a lactation consultant (IBCLC) who met your insurance company's requirements?

6. Did you delay seeing a lactation consultant, or not see one at all, because of issues with insurance?

7. When you saw a lactation consultant, how was billing handled?

8. What was the outcome of your insurance claim for IBCLC services?

9. If a claim was denied, what was the reason given? (check all that apply)

10. Anything else you would like to tell about your experience with insurance coverage for lactation support? 